\title{
Construcción de un modelo de competencias gerenciales para la competitividad de las MYPES ${ }^{1}$
}

Cesatre Chitchoua*

\section{RESUMEN}

El presente trabajo define un modelo de competencias gerenciales para la competitividad de las MYPES en la Alcaldía Cuauhtémoc, Ciudad de México. La investigación tiene un enfoque cuantitativo transversal y un alcance descriptivo; la población son 215 MYPES de la Alcaldía Cuahtémoc, Ciudad de México. Los resultados muestran que el modelo de competencias gerenciales debe considerar las alianzas locales, nacionales e internacionales, renovar constantemente su planta de trabajadores y facilitar la integración de los jóvenes, dados los cambios tecnológicos recurrentes.

Palabras clave: competitividad empresarial, MYPES, organización, permanencia.

Clasificación JEL: M10, M21.

\footnotetext{
1 Esta investigación es producto del Programa Especial con el proyecto "Modelo de competencias gerenciales para la competitividad de las Pymes de la Ciudad de México", financiado por la Secretaría de Investigación y el posgrado del Instituto Politécnico Nacional. La herramienta utilizada en esta investigación fue desarrollada por la doctora Alma Brenda Leyva de la Universidad de Sonora y miembro del Sistema Nacional de Investigadores.

* Profesor-investigador en la Escuela Superior de Economía, sección de Estudios de Posgrado e Investigación, del Instituto Politécnico Nacional, México.

Correo-e: cchiatchoua@ipn.mx
} 


\section{ABSTRACT \\ Construction of a model of managerial competencies for the compe- titiveness of MSEs}

This paper defines a model of managerial competences for the competitiveness of the MYPES in the Cuauhtémoc Mayor's Office, Mexico City. The study takes a cross-sectional quantitative approach and descriptive scope, the population is 215 MYPES of the Cuauhtémoc Mayor's Office, Mexico City. The results show that, given the recurring technological changes, the managerial competences model should consider local, national and international alliances, renew its workforce constantly and facilitate the integration of young people.

Keywords: Business competitiveness, MYPES, organization, permanence.

JEL Clasiffication: M10, M21.

\section{INTRODUCCIÓN}

La dinámica competitiva de los tiempos modernos demanda a la gerencia de las Micro y Pequeñas Empresas (MYPES) contar con competencias adaptadas a la realidad del mercado, que permitan el éxito de sus negocios y el cumplimiento de su misión. Esta gerencia permite contar con personal competente, condición necesaria, aunque no suficiente, para ayudar a que las empresas alcancen sus objetivos y metas.

Para Leyva et al. (2018), las organizaciones del siglo XXI afrontan un entorno dinámico y complejo caracterizado por la incertidumbre, por lo que deben estar preparadas para dar respuestas a los cambios con la finalidad de alcanzar la competitividad empresarial. Al respecto, Drucker (2007) señala que la dinámica competitiva de los actuales escenarios presenta características sumamente relevantes, como la velocidad con que se modifican las condiciones del entorno en el que se mueven las organizaciones, esto es: las nuevas tecnologías, nuevos competidores y clientes más exigentes. Destaca la importancia de contar con un estilo gerencial actual, capaz de interpretar y tomar las acciones con estrategias que contrarresten sus efectos, se aprovechen las oportunidades y se conquisten nuevos mercados.

Es válida la afirmación de Varela (2002) sobre que actualmente las empresas mexicanas, especialmente las MYPES, enfrentan debilidades 
en el logro de su operatividad, debido a la falta de conocimientos y habilidades técnico-administrativas que garanticen competitividad, desarrollo, conquista y permanencia en el mercado. A todo ello se agrega el desconocimiento y la nula importancia que se le ha dado al enorme beneficio de contar con una gerencia por competencias (Leyva et al., 2017).

Por su parte, Ramírez (2005) señala que el reto de la innovación en la actualidad exige de la gerencia aprendizaje y desarrollo de nuevos conocimientos, por lo que las competencias fundamentales para la viabilidad de la gestión empresarial se refieren a la capacidad de la dirección para adquirir conocimiento, adaptarse y cambiar, incluso adelantarse a los cambios. Los actuales mercados globalizados exigen a la gerencia de las MYPES, nuestro caso de estudio, contar con competencias adaptadas al presente les que permitan garantizar el éxito de sus negocios y el cumplimiento de su misión.

De esta realidad no escapan las pequeñas y medianas empresas, en las cuales el papel de los gerentes -como guías empresariales con las competencias gerenciales (conocimientos, habilidades, actitudes e intereses) compatibles con su función- debe orientarse al desarrollo de organizaciones que busquen nuevas fuentes de ventaja competitiva. Por lo anterior, es vital el asesoramiento y capacitación de los gerentes (Melo y Fonseca, 2014).

Sin embargo, sólo algunos investigadores hacen referencia a la importancia del papel de la gerencia en la gestión empresarial de las MYPES y pocos describen la relevancia de las competencias gerenciales para lograr competitividad. En México, el desarrollo económico a mediano y largo plazo debe estar enmarcado en una política industrial cuyo principio fundamental sea el desarrollo de las MYPES, eslabón central y dinamizador del sector industrial y de la actividad económica en general. En este sentido, las competencias gerenciales son un tema poco considerado en México, pero que cada vez interesa más a las empresas que obtienen beneficios gracias al trabajo de gestión de los directivos, los cuales incrementan la competitividad empresarial.

Por lo anterior, vale la pena preguntarse ¿cuál es el modelo de competencias gerenciales que necesitan los gerentes de las MYPES en la Alcaldía Cuauhtémoc, Ciudad de México, en cuanto a sus actividades, requerimientos en materia de gestión y actitudes, conocimiento y habilidades? Por ello, el objetivo de este documento es definir un modelo 
de competencias gerenciales para la competitividad de las MYPES en la Alcaldía Cuauhtémoc. Para lograr el objetivo, el trabajo consta de: introducción, apartado dos, en el que se presenta el marco teórico donde, primero, se enumeran los elementos que integran las competencias gerenciales de las MYPES, y segundo, se analizan las variables a integrar en el modelo basado en competencias para la gestión empresarial de las MYPES en Cuauhtémoc; tercer apartado, donde se determinan los factores integrantes del modelo de gestión por competencias, aplicable en las MYPES según los resultados de campo; cuarto apartado, en el que se discuten los elementos que componen el modelo de competencias gerenciales para la competitividad empresarial en la delegación Cuauhtémoc; y finalmente, conclusión y recomendaciones relevantes.

En la actualidad se requieren gerentes con habilidades para lograr resultados competitivos, establecer excelentes interrelaciones tanto con sus colaboradores, como con los proveedores, los clientes y con todos aquellos que intervienen en la cadena de valor; además, deben poseer conocimientos prácticos en temas económicos, financieros, comerciales, jurídicos, de mercadeo, de gestión humana y dominar dos o más idiomas (Tobón, 2009).

En este sentido, la gerencia por competencias implica la adopción de un sentido que supere el tradicional enfoque del rasgo de personalidad, lo que se fundamenta en una serie de importantes ventajas, tanto para la organización como para la persona (Bateman y Snell, 2005). El concepto de competencia busca, por un lado, una mayor exactitud en las predicciones sobre el rendimiento futuro de las personas y su trabajo, y, por otro, una mayor validez aparente en las técnicas e instrumentos de evaluación para adoptar el concepto de competencia en el mundo empresarial (Armenteros et al., 2008).

Por esta razón surge la necesidad de realizar la presente investigación, que permitirá cumplir y proponer acciones para alcanzar el objetivo de ofrecer a las MYPES información que les permita identificar debilidades, fortalezas, amenazas y oportunidades. Con la ayuda de un Modelo de Gerencia por Competencias podrán enfrentar los cambios de estructura que actualmente requiere este tipo de empresa y obtener resultados de calidad y productividad en sus negocios y que sirva de apoyo a las gerencias de las MYPES. 


\section{MARCO TEÓRICO}

\subsection{Las competencias en las organizaciones}

El término competencia mundial aparece hace más de 20 años para dar cuerpo a la idea del éxito profesional dentro de las organizaciones. En otras palabras, no existe oposición entre aptitudes e inteligencia con competencias, ya que estas cualidades son necesarias para ejercer con éxito la actividad profesional (Alles, 2005).

En la última década, las competencias han adquirido presencia dentro de la gestión de recursos humanos. Tienen antecedentes históricos principalmente en Inglaterra, Estados Unidos, Alemania y Australia (Bateman y Snell, 2005). Las competencias aparecen primeramente relacionadas con los procesos productivos en las empresas, particularmente en el campo tecnológico, en donde el desarrollo del conocimiento ha sido muy acelerado. Por esta razón, se presentó la necesidad de capacitar de manera continua al personal (Benavides, 2001; Koenigsfeld et al., 2012).

Para Solórzano (2008), las competencias gerenciales son aquellas que facilitan la forma de hacer las cosas para solucionar problemas y tomar decisiones dentro del proceso del desarrollo organizacional. Es importante resaltar la asimilación y la acomodación de conocimientos, métodos, técnicas y procedimientos de manera formal e informal, especialmente en situaciones de incertidumbre. Por tanto, las competencias deseadas en un gerente para lograr efectos sobresalientes en la dirección de las empresas deben estar centradas en el ser humano (Lombana et al., 2014).

Esta forma de contemplar las facetas del conocimiento ha dado origen a diversas listas de competencias gerenciales, una de las más populares es la de Levy-Leboyer (2003), quien plantea una serie de competencias universales: presentación oral, comunicación oral, comunicación escrita, análisis de problemas, comprensión de los problemas organizacionales y no organizacionales, planificación, organización, delegación, control, desarrollo de los subordinados, sensibilidad, autoridad sobre individuos, autoridad sobre grupos, tenacidad, negociación, vocación, sentido común, creatividad, toma de riesgos, decisión, entre otros.

Por su parte, Alles (2005) plantea una clasificación basada en los niveles de adquisición de las diferentes competencias en el proceso de 
evolución profesional de los colaboradores de una organización en sus tres niveles: jóvenes sin experiencia laboral, personas con experiencia e historial laboral, y ocupantes de niveles ejecutivos. Esta clasificación está conformada por dos categorías: básicas y diferenciales. En este sentido, Alles ha creído conveniente seleccionar esta clasificación por considerarla pertinente con el contexto de estudio.

- Competencias básicas: conocimientos, habilidades, destrezas y actitudes básicas en el individuo, de cuyo desarrollo generalmente se ocupan los procesos de formación en la sociedad, los cuales resultan preparatorios para su evolución profesional y su desempeño eficaz. Están compuestas por las siguientes categorías: adaptabilidad, flexibilidad, capacidad de aprendizaje, dinamismo, habilidad analítica, iniciativa, modalidades de contacto, responsabilidad, tolerancia a la presión, trabajo en equipo e integridad.

- Competencias diferenciales: distinguen a las personas de niveles superiores de desempeño; pueden desarrollarse al alternar periodos de trabajo y enseñanza, así como en sistemas más informales de aprendizaje organizacional. Al respecto, Alles (2005) presenta una amplia clasificación de estas competencias: colaboración, juicio, empowerment (empoderamiento), nivel de compromiso, orientación al cliente, desarrollo de su equipo, habilidades mediáticas, liderazgo para el cambio, pensamiento estratégico y relaciones públicas.

De acuerdo con Barhem et al. (2011), las competencias gerenciales se reflejan en comportamientos que ciertas personas poseen más que otras y que las convierten en más eficaces en el desempeño de su cargo; son observables en la realidad cotidiana del trabajo o en situaciones de evaluación, en las que aplican de manera integral sus aptitudes, sus rasgos de personalidad y sus conocimientos.

\subsection{Competitividad de las MYPES}

Aunque se calcula que nueve de cada 10 empresas en México entran en la categoría de MYPES y que son responsables de más de $50 \%$ de la economía nacional, alrededor de $80 \%$ muere antes de cumplir su primer año de vida (INEGI, 2010). El por qué del éxito o fracaso de estas empresas exige un análisis más profundo, toda vez que, según datos del INEGI (2020), existen más de 5,487,061 empresas, de las cuales 5,119,909 son micro, lo que equivale a $93.30 \%$ del total; las pequeñas equivalen a $5.42 \%$; las medianas, $0.98 \%$, y las grandes, $0.28 \%$. Estos datos mues- 
tran que las MYPES representan $98.72 \%$ de las empresas nacionales y aportan $64 \%$ de la fuerza laboral que contribuye con $70 \%$ del Producto Interno Bruto (PIB).

A esto hay que sumar el hecho de que $65 \%$ son de carácter familiar; más de $80 \%$ no cuenta con algún tipo de certificación; cerca de $50 \%$ no utiliza técnicas en calidad o productividad; sólo $24 \%$ maneja alguna licencia o patente, y $83 \%$ omite realizar actividad alguna para consolidar su presencia en el exterior. Se debe considerar que la mayoría son empresas de un solo dueño; los locales utilizados son rentados; casi un tercio emplea entre una y dos personas; casi la mitad se financia con recursos de familiares y, en la mayoría de los casos, sus clientes son consumidores locales (INEGI, 2010).

La dinámica competitiva de los actuales escenarios empresariales tiene características relevantes: la velocidad con que se modifican las condiciones del entorno en el que se mueven las organizaciones -nuevas tecnologías, nuevos competidores y clientes más exigentes- demandan un estilo gerencial capaz de interpretarlos, así como el desarrollo de estrategias y acciones que permitan aprovechar las oportunidades para conquistar nuevos mercados (Koenigsfeld et al., 2012).

Actualmente las empresas de México, en especial las MYPES, adolecen de competencias gerenciales en su gestión que les reporten un mejor comportamiento y habilidades más efectivas para realizar cambios en estrategias, programas, estructuras, etc., para una mejor adaptación y para anticiparse a los cambios que pueden afectarles (Longenecker et al., 2009).

\subsection{Las MYPES en la Alcaldía Cuauhtémoc}

La Alcaldía Cuauhtémoc se encuentra ubicada en la región centro del país, conformada por la Ciudad de México, el Estado de México, Hidalgo y Tlaxcala. Constituye el ámbito territorial inmediato de la Zona Metropolitana del Valle de México, integrada por 16 alcaldías de la CDMX, 58 municipios conurbados del Estado de México y uno de Hidalgo. Es importante considerar la visión regional metropolitana en el proceso de planeación, dado que, territorialmente, su crecimiento físico y demográfico ha rebasado sus límites administrativos.

Por lo tanto, es necesario mencionar que todo lo que acontece en la Zona Metropolitana del Valle de México influye en los procesos de desarrollo interno de esta Alcaldía. Localizada en el centro del área urbana 
del Distrito Federal, la Delegación Cuauhtémoc, colinda al norte, con las delegaciones Azcapotzalco y Gustavo A. Madero; al oriente, con la Delegación Venustiano Carranza; al sur, con las delegaciones Benito Juárez e Iztacalco; y al poniente, con la Delegación Miguel Hidalgo (Gobierno de la Ciudad de México, 2018).

La Alcaldía Cuauhtémoc tiene una superficie de $32.44 \mathrm{~km}^{2}$, que representa $2.2 \%$ de la superficie total de la CDMX $\left(1,495.0 \mathrm{~km}^{2}\right)$ y $4.98 \%$ del área urbanizada total de la entidad. En 2015 la población ascendía a 532,553 habitantes, esto es, 5.97\% de la población de la CDMX estimada en 8,918,653 habitantes (INEGI, 2015).

A nivel urbano y por sus características sociodemográficas y de desarrollo económico, la Alcaldía Cuauhtémoc ocupa el segundo lugar en participación del PIB de la ciudad después de la Alcaldía Miguel Hidalgo; concentra un número importante de lo que fue la actividad industrial y comercial histórica del país: $4 \%$ de la producción bruta total nacional, que se estima en 438 mil 606 millones 919 mil pesos. Además, existe una participación económica de 4.5 millones de personas que realizan su actividad laboral o de esparcimiento y actividades culturales; es decir, representa un polo económico de atracción. La Alcaldía concentra más de $80 \%$ de negocios hoteleros de la ciudad y tiene la mayor infraestructura turística, lo cual es una aportación fundamental para el lugar.

Allí se genera $23.8 \%$ del PIB de la CDMX y $4.8 \%$ del nacional por un valor total de casi de 82 mil millones de pesos (a precios de 1993), que representó una riqueza seis veces superior a la de todo el estado de Zacatecas y casi nueve veces superior a la de Tlaxcala, Colima y Nayarit. De esta manera, los vínculos entre las actividades mercantiles, instituciones públicas, privadas, culturales y sociales han hecho posible que esta alcaldía sea la séptima economía del país, con un aporte de $4.8 \%$ del Producto Interno Bruto Neto Nacional, $36 \%$ de equipamiento y $40 \%$ de la infraestructura cultural de toda la CDMX (Gobierno de la Ciudad de México, 2018). 


\section{Metodología}

El enfoque es cuantitativo, descriptivo y transversal, ya que el propósito de la investigación es realizar una propuesta para la implementación de un modelo de competencias gerenciales; el estudio no pretende manipular variables para analizar las consecuencias de esta acción.

\subsection{Selección de la muestra}

El estudio se llevó a cabo en MYPES de la Alcaldía de Cuauhtemoc. Los sujetos de estudio son gerentes de estas empresas. El tipo de la muestra es probabilística, bajo la técnica de muestreo aleatorio simple. La selección de la empresa a encuestar es con base en los listados proporcionados por el Directorio Estadístico Nacional de Unidades Económicas (DENUE) (INEGI, 2019).

De acuerdo con datos del Censo Económico 2019, contenidos en el DENUE, en la Alcaldía Cuauhtémoc existen 75,587 Unidades Económicas, de las cuales 65,737 son MYPES; es decir, tiene entre 0 y 50 trabajadores de todos los sectores económicos. Al analizar la base de datos, se determinó eliminar aquellas empresas que pertenecieran a una institución más grande (franquicias y/o sucursales); grupos de autoayuda -alcohólicos anónimos, capillas y centros religiosos-; centros de salud; gubernamentales; escuelas particulares o públicas; dependencias y oficinas gubernamentales. Se obtuvieron 5,909 empresas. Posteriormente se seleccionaron aquellas pertenecientes a los sectores comercio y servicios, de tal forma que al finalizar el análisis el total de la base sea de 3,900 MYPES (Tabla 1).

TABLA 1

CÁlCULO DEL TAMAÑo DE MUESTRA

\begin{tabular}{cc}
\hline Cuauhtémoc & MYPES \\
\hline Base total & 75,587 \\
\hline Total MYPES & 65,737 \\
\hline MYPES comercio y servicios & 5,909 \\
\hline Depuración & 3,900 \\
\hline
\end{tabular}

Fuente: elaborado con datos del INEGI (2019). 
Para este estudio se utilizó un muestreo aleatorio simple con la siguiente fórmula: Para aplicar la expresión (1) es necesario conocer las proporciones de éxito y fracaso $\mathrm{p}$ y q, cuando éstas son desconocidas; lo usual es fijarlas como: $\mathrm{p}=0.5 \mathrm{y} \mathrm{q}=0.5$. Por otra parte, se requiere conocer el valor de $\alpha$ y por tanto el de $Z_{\propto / 2}$. Los valores usuales para estos parámetros son:

$$
\begin{gathered}
\alpha=0.05, Z_{\propto / 2}=1.96 \text { y } \mathbf{e}=\mathbf{0 . 0 5 .} \\
n=\frac{Z_{\alpha / 2}^{2} N p q}{e^{2} N+Z_{\alpha / 2}^{2} p q}
\end{gathered}
$$

Se obtienen 350 empresas. En el trabajo de campo sólo contestaron 215 empresas, lo que equivale a $61.42 \%$ de tasa de participación.

\subsection{Construcción del instrumento de medición}

El instrumento de medición para obtener estos datos es un cuestionario validado. Se realizó una prueba piloto de la validez interna del instrumento, y posteriormente se procedió al trabajo de campo. Esta herramienta consta de tres bloques: el primero presenta el perfil del ejecutivo; el segundo menciona el perfil de la organización y el tercero define las competencias gerenciales de los ejecutivos.

El primer bloque se enfoca en el Perfil del ejecutivo, esto es, en sus características personales. Se obtienen datos como: género, edad, escolaridad, años de experiencia como gerente, antigüedad en la empresa y puesto. El segundo bloque hace referencia al Perfil de la organización; se cuestiona sobre las características de la organización con preguntas como el giro principal de la empresa, el origen de su capital, la antigüedad de la organización en el mercado y la extensión del mercado donde participa la organización (local, regional, nacional o internacional). Finalmente, el último bloque detalla las Competencias gerenciales: conocimientos, habilidades, destrezas y actitudes de los gerentes en el desarrollo de sus funciones diarias en la organización; se da la opción de contestar de 1 a 5 .

Tomando en cuenta la muestra del estudio, se evaluó la confiabilidad con la aplicación del alfa de Cronbach para la encuesta sobre las competencias gerenciales de los directivos. Los resultados de confiabilidad obtenidos se muestran en la Tabla 2; se observa que la fiabilidad de los reactivos propuestos en el instrumento de medición tienen una 
correlación extremadamente alta, ya que la implementación del alfa de Cronbach arroja resultados bajo un rango estandarizado aceptado, el cual se encuentra entendido entre 0.8 y 0.55 , y cualquier resultado que se enfoque dentro de esos números es considerado representativo de manera porcentual; lo anterior indica que la congruencia de las preguntas permite favorecer su procesamiento en el software estadístico.

TABLA 2

ALFA DE CRONBACH

\begin{tabular}{ccc}
\hline Dimensión & Alfa de Cronbach & Número de Elementos \\
\hline Perfil del ejecutivo & 0.765 & 6 \\
\hline Perfil de la organización & 0.749 & 4 \\
\hline Competencias gerenciales & 0.758 & 6 \\
\hline Depuración & 3,900 & \\
\hline
\end{tabular}

Fuente: elaboración propia.

El instrumento se aplicó en junio y agosto de 2019; una vez realizadas las encuestas, se integraron en una base de datos para su posterior procesamiento en el Paquete Estadístico Excel.

\section{RESULTADOS Y DISCUSIÓN}

En ese apartado se presentan los resultados del trabajo de campo realizado en 215 empresas. Esencialmente son micro y pequeñas empresas del sector de los servicios. En este trabajo se consideraron aquellas que desearon participar en el estudio. Cabe mencionar que la integración de empresas de mayor tamaño resulta cada vez más dificil.

\subsection{Perfil del ejecutivo}

En esta sección se describen género, edad, escolaridad, experiencia, antigüedad y puesto del encuestado. La Figura 1 muestra el género de los diferentes participantes. La mayoría de los encuestados es de sexo masculino; es decir, $51 \%$ son hombres, mientras que $49 \%$ son mujeres. La diferencia es mínima, lo que indica que la incorporación de la mujer en el mercado laboral es cada más efectiva. 


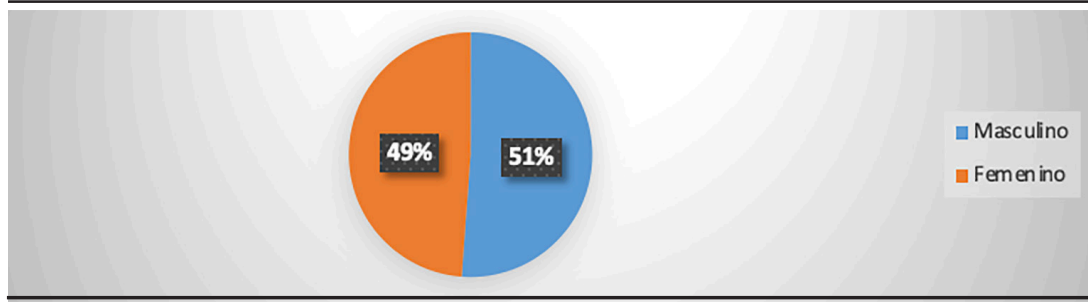

Fuente: elaborado con los resultados obtenidos de la encuesta "Competencias gerenciales".

La Figura 2 muestra la edad de los participantes: 35\% tiene menos de 25 años, y $51 \%$ tiene entre 26 y 45 años. El promedio de edad es relativamente joven; la mayor proporción se encuentra en la generación millenial. 14\% restante tiene más de 45 años. Este resultado muestra la necesidad de los empresarios de renovar constantemente su planta de trabajadores e integrar jóvenes en el mundo del trabajo.

\section{FIGURA 2}

EDAD

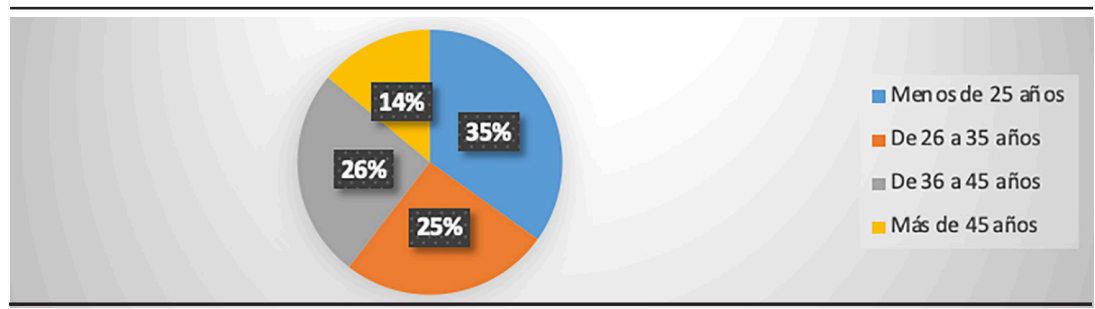

Fuente: elaborado con los resultados obtenidos de la encuesta "Competencias gerenciales".

La Figura 3 describe el nivel de escolaridad de los encuestados. Se observa que $63 \%$ de los participantes no tiene una licenciatura. No es sorprendente, dado que la mayoría de las empresas encuestadas son micro y pequeñas empresas. Se evidencia que la mayoría de los egresados universitarios buscan trabajos en empresas de tamaño mediano y grande, lo que implica mayor estabilidad y mayor remuneración. Al respecto, Kane y Rouse (1993) identifican que, por cada año adicional, un individuo con formación universitaria gana $5 \%$ más que alguien que únicamente completó el bachillerato. Asimismo, Rojas et al. (2000) afirman que cuando una persona tiene mayor grado de educación, su 
ingreso salarial aumenta, mientras que un universitario graduado recibe $78 \%$ más de salario que una persona sin estudios (Sáenz y Ávila, 2016).

Por otro lado, 29\% tiene nivel licenciatura, lo cual, para Hernández (2012) implica que les falta valor agregado, a pesar de las oportunidades de trabajo, por lo que los profesionistas optan por empleos no calificados, ya que es verdaderamente difícil cubrir el perfil requerido. Finalmente, $6 \%$ tiene nivel licenciatura más una especialidad.

FIGURA 3

ESCOLARIDAD

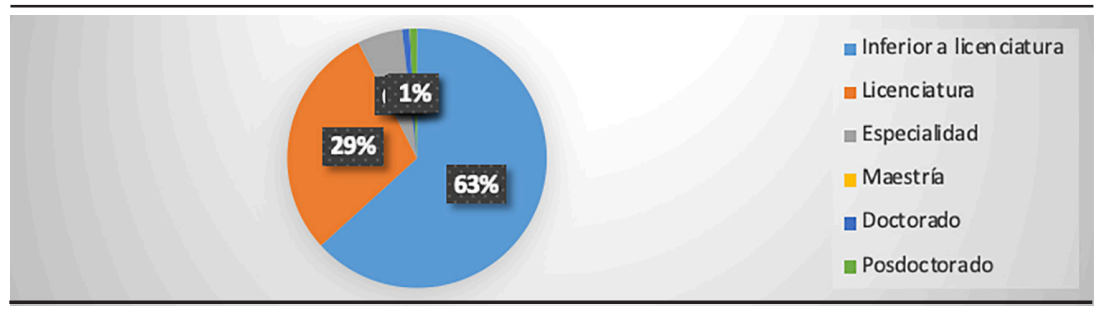

Fuente: elaborado con los resultados obtenidos de la encuesta "Competencias gerenciales".

La Figura 4 muestra el nivel de experiencia de los participantes. Se observa que $61 \%$ de los encuestados tiene menos de 5 años, lo que representa poco tiempo; 25\% tiene entre 6 y 10 años, y 14\% tiene 15 años o más. Una de las debilidades de las micro y pequeñas empresas es la alta rotación de su personal dada la informalidad de su estructura. Para González (2006) las causas de la rotación del personal son: enfermedades crónicas, accidentes que producen lesiones parciales o totales permanentes, jubilación o muerte. Entre las segundas causas se encuentran la insatisfacción, bajos sueldos, mala integración del trabajador a la organización, falta de identificación del empleado con los objetivos de la organización, mala selección del personal y falta de movilidad interna (programa de ascensos y traslados). Por su parte, las terceras son generadas por ascensos, promociones y traslados. 


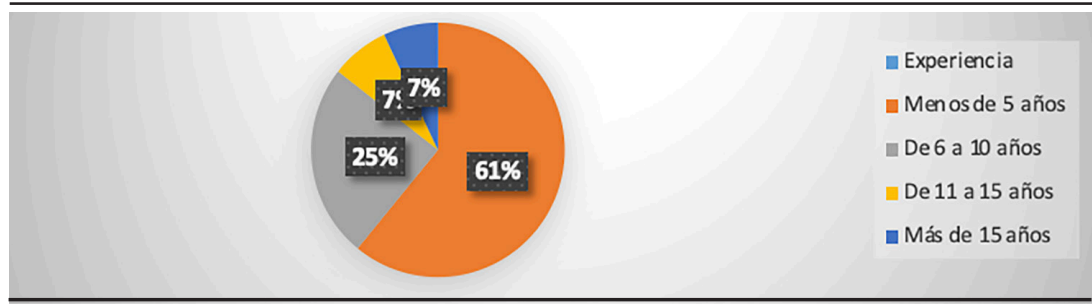

Fuente: elaborado con los resultados obtenidos de la encuesta "Competencias gerenciales".

La Figura 5 describe la antigüedad de las empresas participantes. Se percibe que $67 \%$ de las empresas encuestadas tiene menos de 5 años en el mercado, tiempo de permanencia promedio de las micro y pequeñas empresas; mientras que 19\% tiene entre 6 y 10 años de vida en el mercado. Finalmente, 14\% tiene de 11 a 15 años o más.

\section{FIGURA 5}

ANTIGÜEDAD

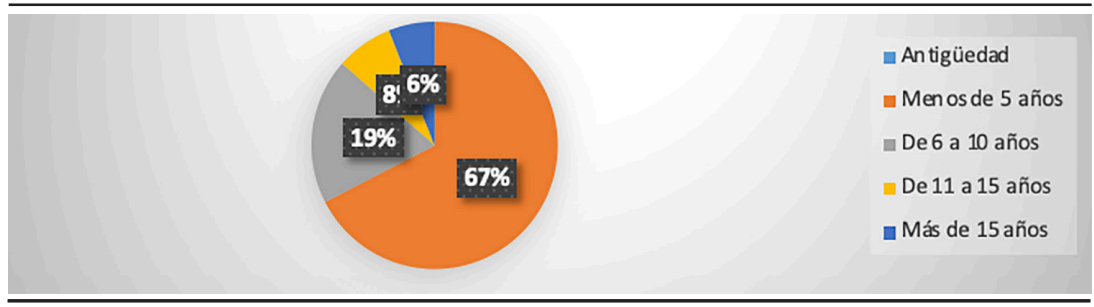

Fuente: elaborado con los resultados obtenidos de la encuesta "Competencias gerenciales".

La Figura 6 presenta el puesto que ocupa el participante. Los resultados muestran que $67 \%$ de los encuestados son gerentes de la empresa; en este estudio se les denomina "encargado" o "director", mientras que $26 \%$ corresponde a despachador, cajero, auxiliar administrativo o técnico. Finalmente, $6 \%$ representa el contador, y $1 \%$, el dueño. 


\section{FIGURA 6}

PUESTO

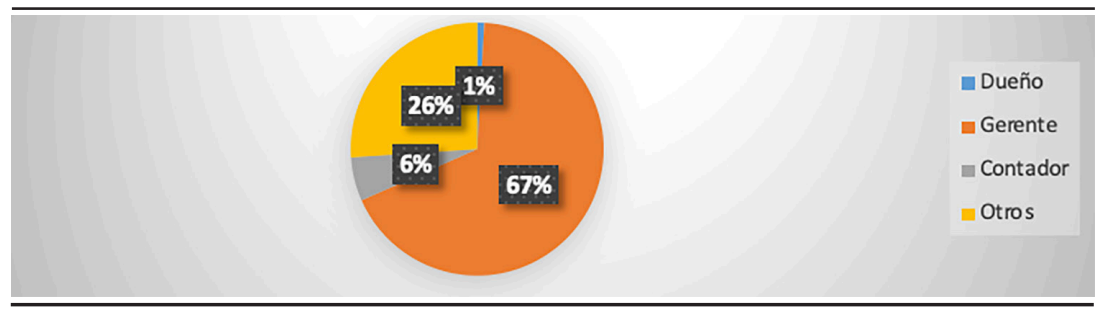

Fuente: elaborado con los resultados obtenidos de la encuesta "Competencias gerenciales".

\subsection{Perfil de la organización}

En esta sección se detallan el giro, origen del capital, permanencia y mercado de atención. Es importante enfatizar que no se seleccionaron a priori las empresas participantes, sino que únicamente contestaron aquellas que por voluntad propia aceptaron colaborar en el estudio. La Figura 7 muestra que $76 \%$ de las empresas están orientadas al sector comercio, $15 \%$, industria, y $9 \%$, servicios. Cabe señalar que no hay empresas del sector agropecuario.

FIGURA 7

GIRO

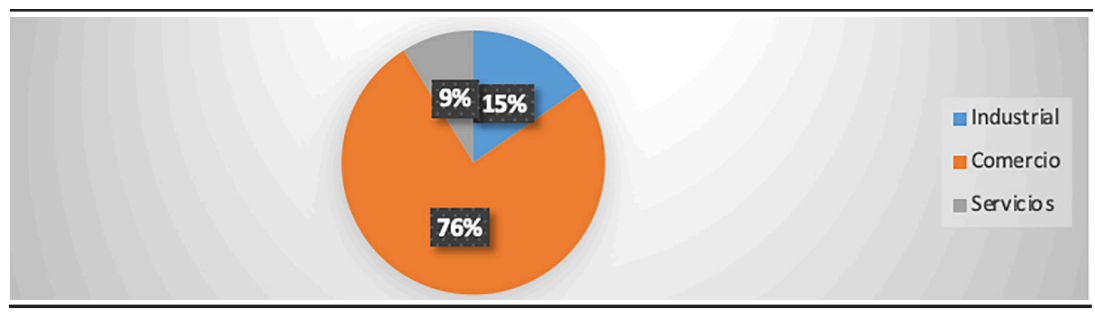

Fuente: elaborado con los resultados obtenidos de la encuesta "Competencias gerenciales".

La Figura 8 describe el origen del capital; 97\% es nacional; es decir, los dueños de las empresas son mexicanos, mientras que $1 \%$ representa la participación de extranjeros. Es importante mencionar que muchos extranjeros se han naturalizado por cuestiones profesionales o administrativas y son considerados nacionales, razón por la cual la casi totalidad del capital está en el interior del país. 
FIGURA 8

ORIGEN DEL CAPITAL

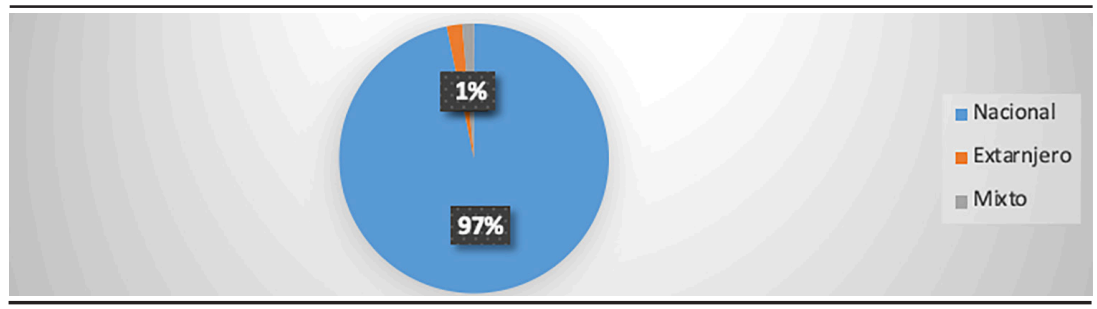

Fuente: elaborado con los resultados obtenidos de la encuesta "Competencias gerenciales".

La Figura 9 describe la permanencia de las empresas. Destaca que 68\% de las empresas tiene menos de 5 años de existencia en el mercado; este resultado no es sorprendente dado que la mayoría de las MYPES no logran permanecer en el mercado porque buscan enfocar su éxito únicamente a la reducción de gastos, la calidad o el servicio, y no a reducir sus costos o incrementar sus ventas. Al respecto, Puértolas (2016) afirma que las PYMES en México carecen de competitividad; un dato importante es que en los últimos cinco años, tres de cada 10 empresas cerraron.

FIGURA 9

PERMANENCIA DE LA EMPRESA

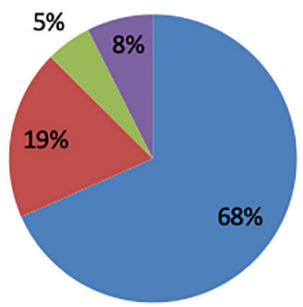

Menos de 5 años

घ De 6 a 10 años

$\square$ De 11 a 15 años

口 Más de 15 años

Fuente: elaborado con los resultados obtenidos de la encuesta "Competencias gerenciales".

La Figura 10 presenta el mercado de atención de las empresas. $68 \%$ tiene presencia de menos de 5 años en su sector de competencia; $19 \%$, entre 6 y 10 años, y 13\% entre 15 y más años en el mercado. 
FIGURA 10

MERCADO DE ATENCIÓN

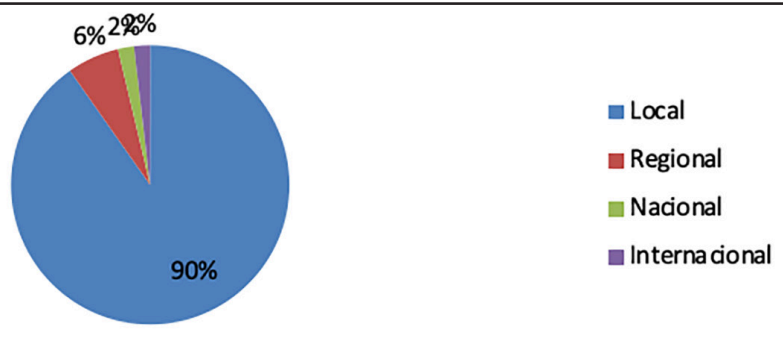

Fuente: elaborado con los resultados obtenidos de la encuesta "Competencias gerenciales".

\subsection{Competencias gerenciales}

En esta sección se describen las competencias desarrolladas por los responsables de las empresas. La Figura 11 muestra la Organización y gestión empresarial. Se midieron nueve indicadores relacionados con los conocimientos, habilidades, destrezas y aptitudes del empresario. Los resultados muestran que el empresario cuenta y aplica parcialmente la competencia de identificación y resolución de problemas, planteamiento de soluciones y resolución de diferencias de ideas $\mathrm{u}$ opiniones de las partes, y encuentro de las causas y soluciones de una situación, así como el análisis, organización y presentación de información a través de datos numéricos.

En relación con los cinco indicadores restantes, los resultados muestran que el empresario cuenta con las destrezas, conocimientos y habilidades, y aplica la competencia en gran medida en la comunicación con diferentes personas; supera las expectativas del cliente en la identificación y solución de problemas, escucha y percibe las respuestas y opiniones de los demás, resuelve eficazmente las situaciones que pueden afectar a las relaciones entre personas y la empresa, y llega a acuerdos satisfactorios para todos. 
FIGURA 11

ORGANIZACIÓN Y GESTIÓN

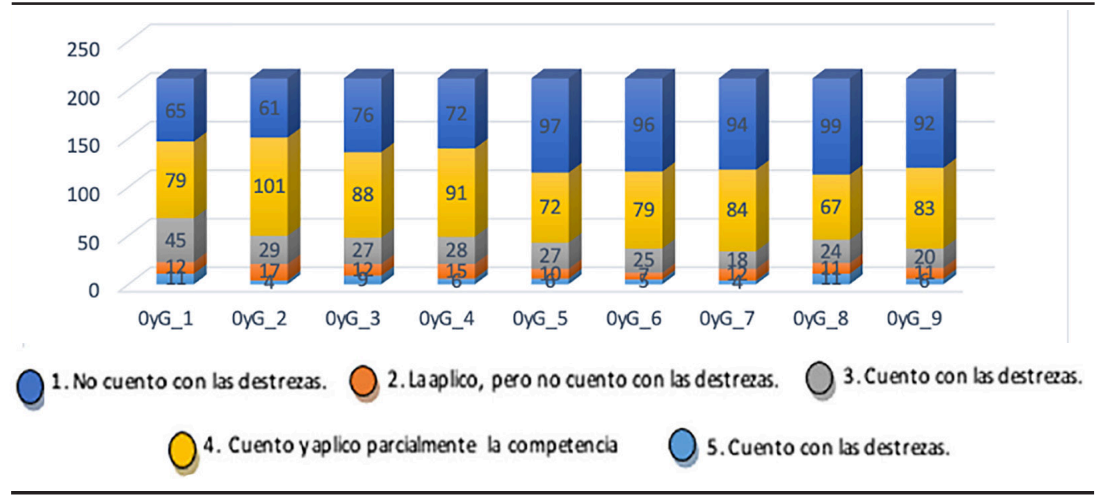

Fuente: elaborado con los resultados obtenidos de la encuesta "Competencias gerenciales".

La Figura 12 muestra la administración estratégica. Se midieron siete indicadores relacionados con los conocimientos, habilidades, destrezas y aptitudes del empresario. Los resultados muestran que el empresario cuenta $\mathrm{y}$ aplica parcialmente la competencia respecto a plantear soluciones y resolver diferencias de ideas u opiniones de las partes, encontrar las causas y soluciones de una situación o problema específico, cuyos resultados son sustentados con eficacia y confiabilidad, así como manejar técnicas para formular problemas.

En cuanto a los cuatro indicadores restantes, los resultados muestran que el empresario cuenta con las destrezas, conocimientos y habilidades y las aplica en gran medida para la búsqueda constante de oportunidades en el entorno para garantizar la viabilidad de los objetivos empresariales, y genera entre sus colaboradores el mismo espíritu gestor de nuevas acciones; manifiesta dinamismo orientado hacia la consecución de un objetivo particular; busca constantemente oportunidades en el entorno y las capitaliza en nuevos proyectos; aunado al correcto manejo y control financiero de la empresa. 
FIGURA 12

ADMINISTRACIÓN ESTRATÉGICA

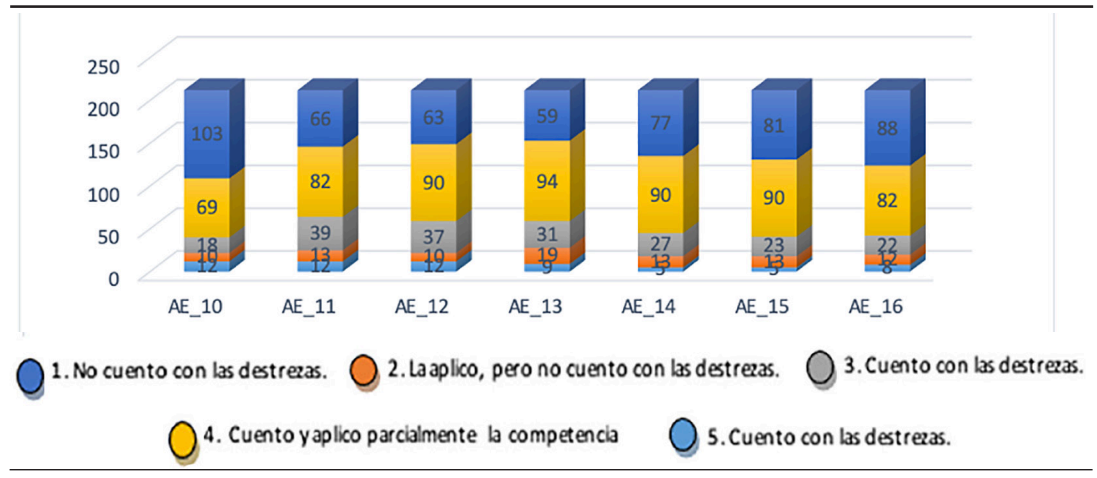

Fuente: elaborado con los resultados obtenidos de la encuesta "Competencias gerenciales".

La Figura 13 describe la Planeación Estratégica. Se utilizaron ocho indicadores y los resultados muestran que el empresario cuenta con las destrezas, conocimientos y habilidades y las aplica en gran medida para elaborar planes de acción, incluyendo los recursos necesarios y los sistemas de control; identifica las tendencias del medio; detecta el nivel de demanda de productos similares en el mercado; detecta riesgos y/o oportunidades en las acciones empresariales; presupuesta los recursos necesarios para alcanzar los objetivos de la empresa; establece tácticas y estrategias; detecta puntos claves de la empresa que afectan la competitividad y evalúa información.

FIGURA 13

PLANEACIÓN ESTRATÉGICA

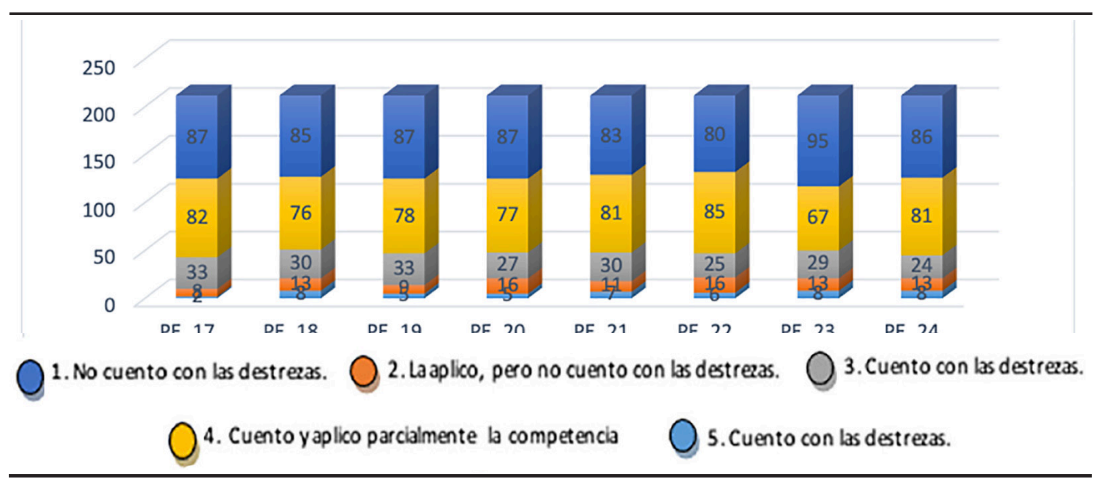

Fuente: elaborado con los resultados obtenidos de la encuesta "Competencias gerenciales". 
La Figura 14 describe la Globalización; para ello se utilizaron cinco indicadores. Los resultados indican que el empresario cuenta con las destrezas, conocimientos y habilidades y las aplica en gran medida para adaptarse a entornos cambiantes; identifica y aprovecha oportunidades de innovación tecnológica; identifica el impacto de la globalización en el contexto económico, tecnológico y social de la empresa. Los resultados restantes muestran que el empresario cuenta y aplica parcialmente la competencia respecto a simular escenarios de actuación en mercados que representen una ventaja competitiva y amplios conocimientos, habilidades, destrezas y actitudes de la competencia mencionada.

FIGURA 14

GLOBALIZACIÓN

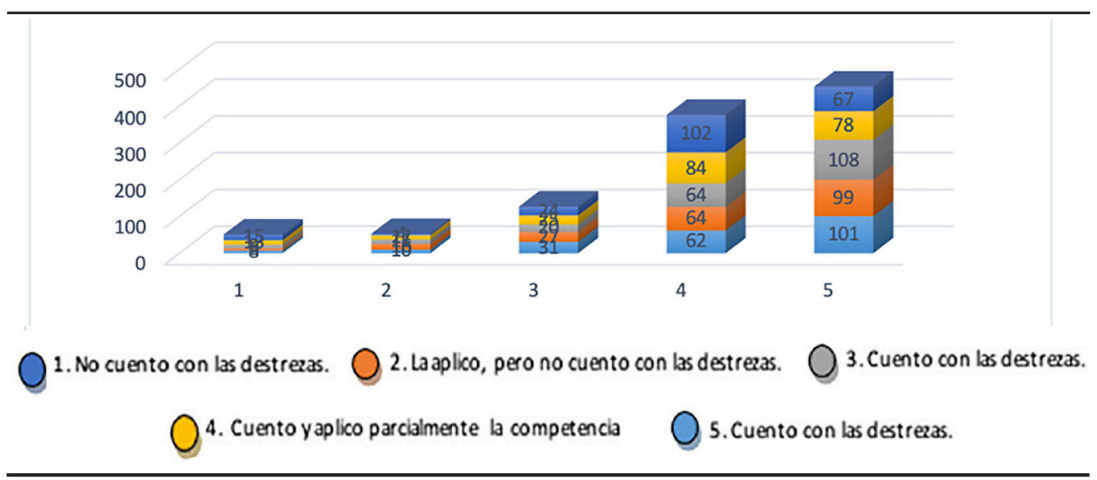

Fuente: elaborado con los resultados obtenidos de la encuesta "Competencias gerenciales".

La Figura 15 presenta los Recursos humanos dentro de la organización. Los participantes afirmaron que el empresario cuenta y aplica parcialmente la competencia en torno a anteponer el bien colectivo sobre los intereses particulares; genera oportunidades de desarrollo y analiza situaciones desde diversas perspectivas. Por otro lado, los encuestados aseveran que el empresario cuenta con las destrezas, conocimientos y habilidades y las aplica en gran medida para construir relaciones de confianza, integrarse e integrar equipos de trabajo efectivos; propicia la participación de su grupo de trabajo; realiza y promueve el trabajo en equipo; evalúa y retroalimenta a sus colaboradores; genera un ambiente de motivación y compromiso en el equipo de trabajo, y fomenta el aprendizaje y la formación a largo plazo. 
FIGURA 15

RECURSOS HUMANOS

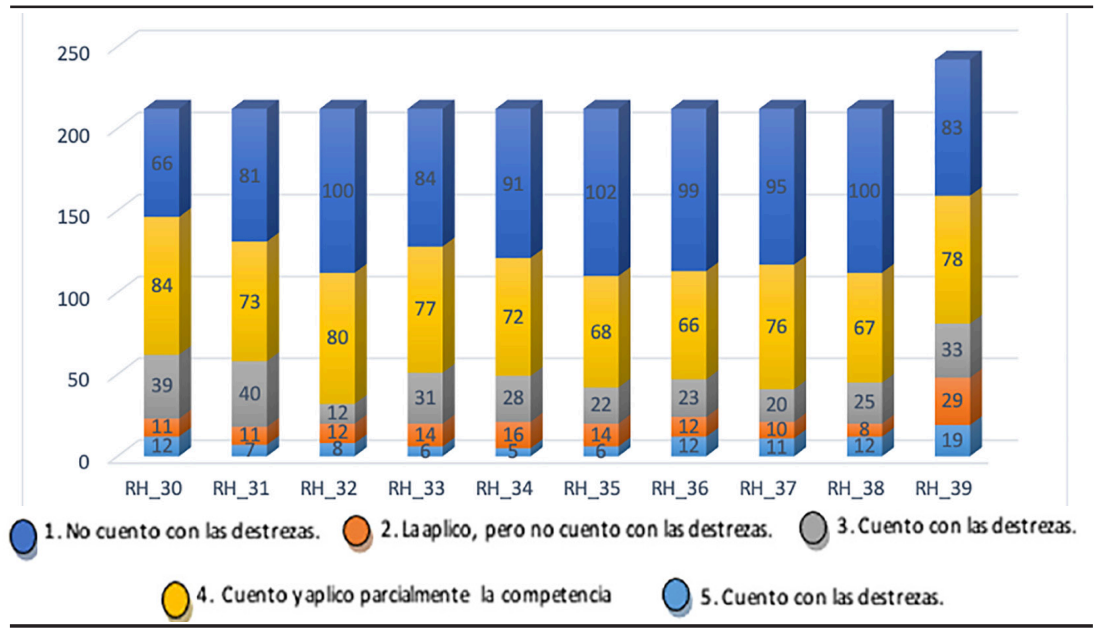

Fuente: elaborado con los resultados obtenidos de la encuesta "Competencias gerenciales".

Finalmente, la Figura 16 detalla la Competitividad empresarial. Mediante el uso de cinco indicadores, los participantes expresaron que el empresario cuenta y aplica parcialmente la competencia relativa a la identificación de las variables, construcción de modelos y diseño de estrategias para mejorar la competitividad de la empresa. También, afirmaron que el empresario cuenta con las destrezas, conocimientos y habilidades y las aplica en gran medida para construir un sistema de seguimiento que ayude a lograr de forma efectiva la competitividad de la empresa, identificar empresas relacionadas y de soporte, adquirir ventajas competitivas a través de una cadena de valor dentro de la empresa y detectar mejores formas de competir, innovando. 
FIGURA 16

COMPETITIVIDAD EMPRESARIAL

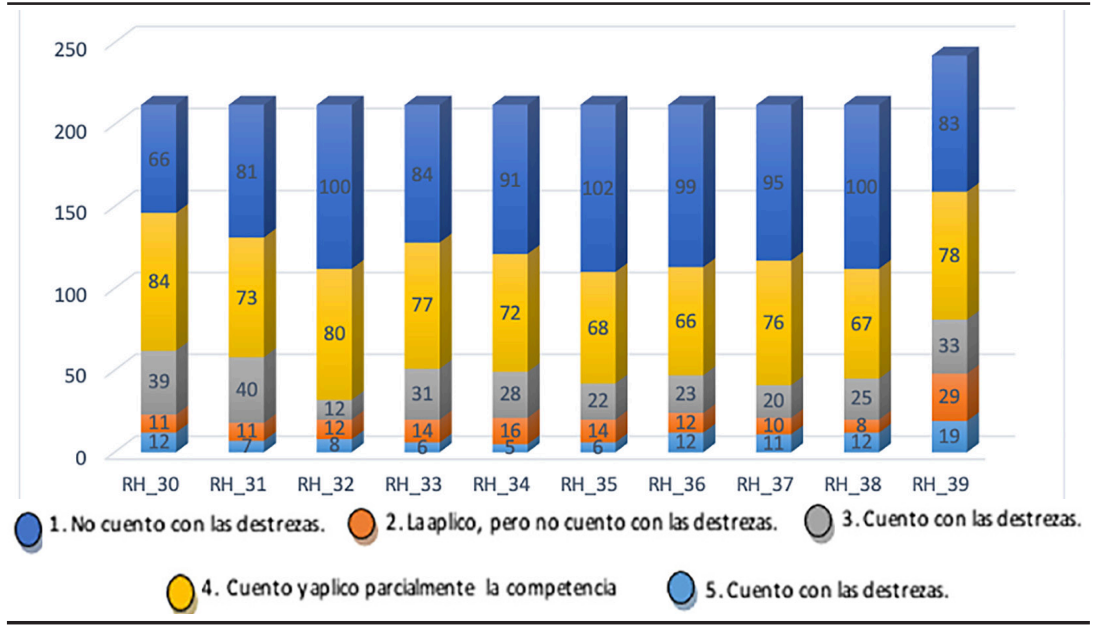

Fuente: elaborado con los resultados obtenidos de la encuesta "Competencias gerenciales".

\section{Discusión}

Los resultados de este estudio muestran que el modelo de competencias gerenciales debe considerar los siguientes elementos: el perfil del ejecutivo debe ser de sexo indistinto; tener menos de 25 años de edad; preparatoria concluida y con aspiración a estudios universitarios o cursos de capacitación en técnicas administrativas, y experiencia menor a 5 años, dados los sueldos bajos en las empresas de pequeño tamaño.

Este resultado es coherente con lo propuesto por Mujica, quien asevera que los requisitos para ocupar cargos ejecutivos en las empresas han ido evolucionando radicalmente, especialmente en los últimos años (2018: 105). Ello tiene suma importancia a la hora de definir el contenido de los programas de educación superior para las carreras profesionales.

\subsection{La educación superior y el mercado laboral}

De igual manera, el perfil requerido del gerente es de director o encargado en una empresa con menos de cinco años de presencia en el mercado. Respecto al perfil de la organización, el sector de comercio es el más apreciado, con capital principalmente nacional y una permanencia y presencia de menos de cinco años en su sector de competencia. 
En cuanto a la organización y gestión, las competencias del gerente deben estar enfocadas en las destrezas, conocimientos y habilidades, especialmente en las Tecnologías de Información y Comunicación (TIC) (Cano y Polanco, 2018; Koenigsfeld et al., 2012), aplicadas en gran medida para comunicarse con diferentes personas, superar las expectativas del cliente en la identificación y solución de problemas, escuchar y percibir las respuestas y opiniones de los demás, resolver eficazmente las situaciones que pueden afectar las relaciones entre personas y la empresa, y llegar a acuerdos satisfactorios para todos.

Respecto a la competencia gerencial sobre Administración estratégica, el gerente cuenta con las destrezas, conocimientos y habilidades, y las aplica en gran medida para la búsqueda constante de oportunidades en el entorno y para garantizar la viabilidad de los objetivos empresariales, con lo que genera en sus colaboradores el mismo espíritu gestor de nuevas acciones. Manifiesta dinamismo orientado a la consecución de un objetivo particular; busca constantemente oportunidades en el entorno y las capitaliza en nuevos proyectos, además de que tiene un adecuado manejo y control financiero de la empresa.

Asimismo, en la competencia Planeación estratégica, el gerente cuenta con las destrezas, conocimientos y habilidades, y las aplica en gran medida para elaborar planes de acción, incluyendo los recursos necesarios y los sistemas de control; identifica las tendencias del medio; detecta el nivel de demanda de productos similares en el mercado, así como los riesgos y/o oportunidades en las acciones empresariales. Presupuesta los recursos necesarios para alcanzar los objetivos de la empresa; establece tácticas y estrategias; detecta puntos claves de la empresa que afectan la competitividad y evalúa información.

En lo concerniente a la Globalización, el gerente cuenta con las destrezas, conocimientos y habilidades, y las aplica en gran medida para adaptarse a entornos cambiantes; identifica y aprovecha oportunidades de innovación tecnológica e identifica el impacto de la globalización en el contexto económico, tecnológico y social de la empresa. En cuanto a los Recursos humanos, el encargado cuenta con las destrezas, conocimientos y habilidades y las aplica en gran medida para construir relaciones de confianza; se integra e integra equipos de trabajo efectivos; propicia la participación de su grupo de trabajo; realiza y promueve el trabajo en equipo; evalúa y retroalimenta a sus colaboradores; genera en el 
equipo de trabajo un ambiente de motivación y compromiso, y fomenta el aprendizaje y la formación a largo plazo (Melo y Fonseca, 2014).

Respecto a la Competitividad empresarial, el director posee las destrezas, conocimientos y habilidades y las aplica en gran medida para construir un sistema de seguimiento que ayude a lograr, de forma efectiva, la competitividad de la empresa; identifica empresas relacionadas y de soporte; adquiere ventajas competitivas a través de una cadena de valor dentro de la empresa y detecta mejores formas de competir a través de la innovación (Leyva et al., 2018).

FIGURA 17

MODELO DE COMPETENCIAS GERENCIALES

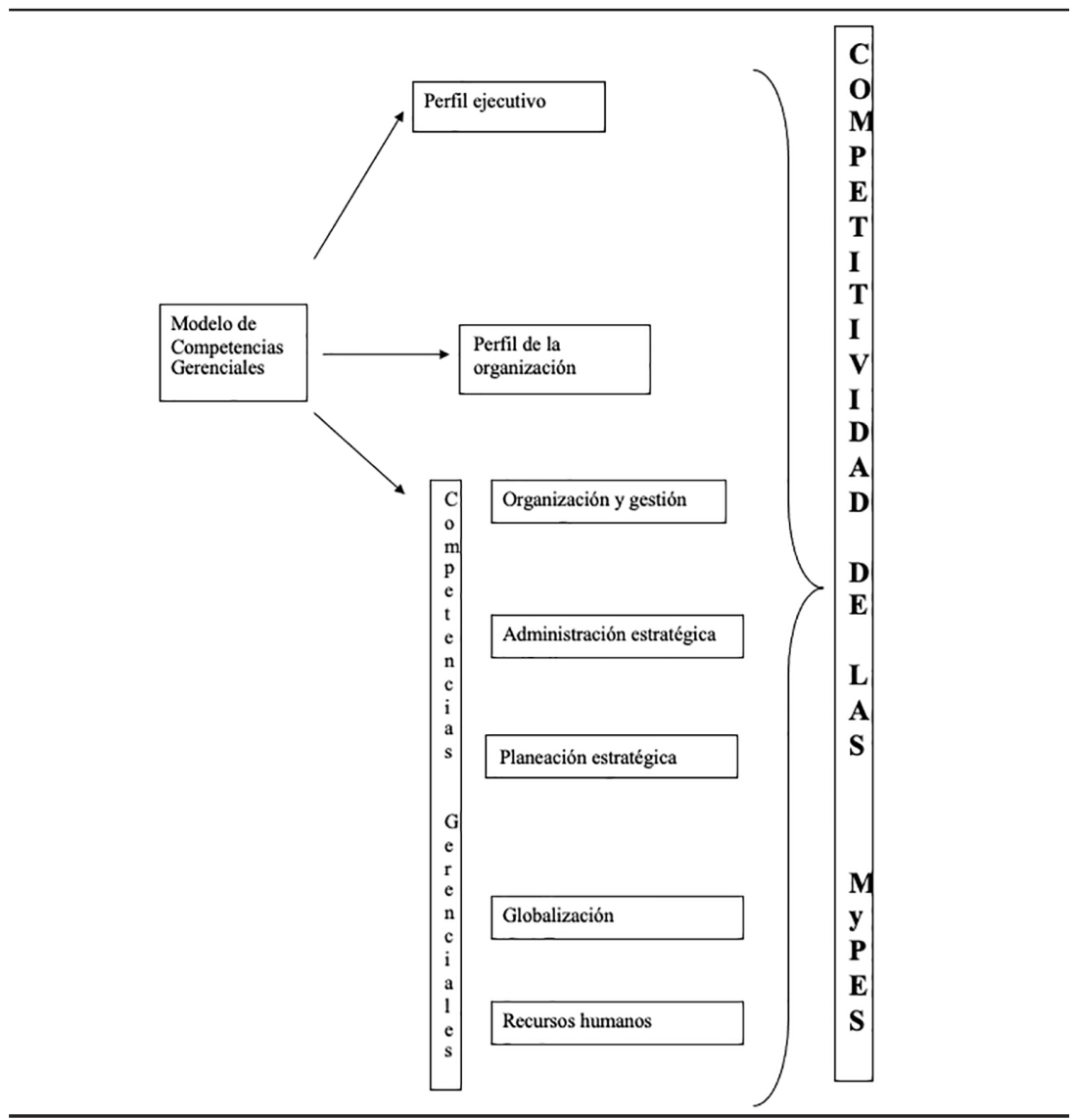

Fuente: elaboración propia. 


\section{CONCLUSIÓN}

La realidad de las MYPES centra su atención de largo plazo en una gestión humana que, teniendo en cuenta su desempeño distintivo, sea capaz de impulsar el desarrollo de los sectores productivos donde están inmersas; por ello se convierte en fuente de ventaja competitiva para la toma de decisiones y para potenciar la diferenciación en el mercado.

Los resultados de este estudio muestran que quienes asumen el cargo de administrar las empresas son cada vez más jóvenes, con un nivel de estudio apenas universitario y un nivel de experiencia menor a cinco años. En el ámbito empresarial, los gerentes cuentan con amplia habilidad para comunicar tanto dentro de la empresa como con su entorno, dado el desarrollo de los medios digitales disponibles. De la misma manera, cuentan con destrezas para desarrollar habilidades directivas, como trabajo en equipo y liderazgo. Finalmente, la capacitación y formación son elementos esenciales para fomentar la innovación y el control del desarrollo tecnológico.

Sin embargo, estas competencias no se pueden considerar como un hecho para todas las empresas de la Alcaldía Cuauhtémoc, como lo mencionan Walo (2000) y Mc Gregor et al. (2004), dado que las competencias que necesitan desarrollar los gerentes en este siglo no son las que están escritas en los libros de texto. Al respecto, Barhem et al. (2011) argumentan que el manejo de las competencias no se puede percibir como un sistema lógico, sino como un manejo holístico, dado el carácter dinámico y cada vez más complejo del entorno.

Este estudio ha permitido observar que la inclusión de la mujer en el ámbito laboral cada vez es más creciente y que los jóvenes son mayormente solicitados en el mercado del empleo, dadas sus capacidades en el manejo de las nuevas herramientas tecnológicas y su entendimiento del entorno complejo y cada vez más globalizado. Asimismo, este análisis ha mostrado que no se necesitan altos niveles de estudios para administrar las empresas, dado que son MYPES, donde los salarios son bajos y los gerentes tienen poca experiencia. Sobre ese tema, Coque et al. (2013) mencionan que la universidad aparece como un elemento de apoyo a través de asignaturas de creación de empresas, servicios -como asesoría, publicidad o contactos- y el conocimiento mutuo de futuros socios. Sin embargo, su trabajo revela que aún queda mucho 
camino por recorrer en las instituciones de nivel superior para lograr una promoción más eficaz.

La presente investigación tiene gran relevancia para el país dada su importancia tanto en la creación de empleo como en el crecimiento de la economía; por lo tanto, en el ambiente económico global en el que se desempeñan las organizaciones es indispensable generar alianzas con empresas extranjeras para atraer capital que fortalezca el crecimiento y el desarrollo de los negocios, así como para fomentar actividades de investigación e innovación. Además, el gerente debe poseer habilidades de liderazgo para identificar problemas y aportar soluciones inmediatas, así como desarrollar estrategias de diseño para mejorar la competencia de la empresa.

Finalmente, con los resultados aquí presentados se evidencia que existen áreas de oportunidad en el modelo de competencia gerencial de los directivos para la competitividad de las MYPES en la Alcaldía Cuauhtémoc, por lo que es necesario desarrollar dentro de las PYMES un ambiente favorable para la construcción de las habilidades de los gerentes. Por tanto, las pequeñas empresas deberán renovar constantemente su planta de trabajadores y facilitar la integración de los jóvenes, dados los cambios tecnológicos recurrentes. Igualmente, el tema de la competitividad de los salarios cobra relevancia para la permanencia de las MYPES, de allí la necesidad de desarrollar un marco regulatorio (fiscal, legal y de seguridad social) para la permanencia de este tipo de empresas. 


\section{BIBLIOGRAFÍA}

Alles, Martha (2005), Dirección estratégica de recursos humanos. Gestión por competencias, Ediciones Granica, S.A. Argentina.

Armenteros, María, Nancy García y Ricardo Negrin (2008), "Innovación educativa en la formación de directivos. Experiencias desde un enfoque basado en competencias", Journals Ingeniería Industrial-DOAJ-, 29(1), pp. 34-41.

Barhem, Belal, Hassan Younies y Pamela Smith (2011), "Ranking the future global manager characteristics and knowledge requirements according to UAE business managers opinions", Education, Business and Society, Contemporary Middle Eastern Issues, 4(3), pp. 229-247.

Bateman, Thomas y Scott Snell (2005), Administración: una ventaja competitiva, McGraw Hill Interamericana, México, $4^{\mathrm{a}}$ ed.

Benavides Espindola, Olga (2001), Competencias y Competitividad. Diseño para organizaciones Latinoamericanas. Mc Graw Hill, México, Distrito Federal.

Cano Martínez, Jeimy José y Erika Polanco Chamorro (2018), "Reflexiones sobre la evolución del rol del ejecutivo de ti 1960-2017", Encuentro internacional de investigadores en administración, Universidad Externado de Colombia, Colombia.

Coque Martínez, Jorge, Francisco Díaz Bretones y Nuria López Mielgo (2013), "Factores para la puesta en marcha y el éxito de microempresas asociativas creadas por jóvenes egresados universitarios revesco", Revista de Estudios Cooperativos, 112, pp. 66-94.

Drucker, Peter (2007), La Gerencia para el cambio, Norma, Madrid.

Gobierno de la Ciudad de México (2018), "Programa Delegacional de Desarrollo en Cuauhtémoc, 2016-2018”, Delegación Cuauhtémoc, Ciudad de México. <http://www.cuauhtemoc.cdmx.gob.mx/static/ls/2017/03/15/ Programa_de_Desarrollo_Delegacional_2016-2018_1.pdf $>$.

González M. (2006), La rotación de personal como elemento laboral que afecta la misión de la organización. Tesis. Universidad Autónoma de Nuevo León. Disponible en: https://core.ac.uk/download/pdf/76584789.pdf

Hernández, Lilian (2012), "Sin ejercer, 60\% de profesionistas; egresan con conocimientos obsoletos". Excélsior. <https://www.excelsior.com. $\mathrm{mx} / 2012 / 07 / 30 /$ nacional/850633>.

Instituto Nacional de Estadística y Geografía (INEGI) (2020), “Directorio Estadístico Nacional de Unidades Económicas". <https://www.inegi.org.mx/ app/mapa/denue/Default.aspx?idee $=572850>$.

Instituto Nacional de Estadística y Geografía (INEGI) (2019), “Directorio Estadístico Nacional de Unidades Económicas", Delegación Cuauhtémoc.

Instituto Nacional de Estadística y Geografía (INEGI) (2015), "Encuesta Intercensal." $<$ http://cuentame.inegi.org.mx/poblacion/habitantes.aspx?tema $=\mathrm{P}>$ 
Instituto Nacional de Estadística y Geografía (INEGI), (2010), Resumen de los resultados de los censos económicos 2009. México: INEGI.

Kane, Thomas y Cecilia Rouse (1993), "Labor Market Returns to Two- And Four-Year College: Is a Credit a Credit And Do Degrees Matter?”, Working Paper núm. 690, Princeton University, Department of Economics, Industrial Relations Section.

Koenigsfeld, Jason Paul, Hyewon Youn, Joe Perdue y Robert Woods (2012), "Revised competencies for private club managers", International Journal of Contemporary Hospitality Management, 24(7), pp. 1066-1087.

Lévy-Leboyer, Claude (2003), Gestión de las competencias, Ediciones Gestión 2000, S.A., Barcelona.

Leyva Carreras, Alma Brenda, Cavazos Arroyo, Judith y Espejel Blanco Joel Enrique (2018), "Influencia de la planeación estratégica y habilidades gerenciales como factores internos de la competitividad empresarial de las Pyme", Contaduría y Administración, 63 (3), pp. 1-21.

Leyva Carreras, Alma Brenda, Joel Enrique Espejel Blanco y Judith Cavazos Arroyo (2017), "Habilidades gerencial es como estrategia de competitividad empresarial en las pequeñas y medianas empresas (Pymes)", Perspectiva Empresarial, 4(1), pp. 7-22.

Lombana, Jahir, Leonor Cabeza, Jaime Castrillón y Álvaro Zapata (2014), "Formación en competencias gerenciales. Una mirada desde los fundamentos filosóficos de la administración", Estudios Gerenciales 30, pp. 301-313.<http://dx.doi.org/10.16967/rpe.v4n1a1>.

Longenecker, Justin, Carlos Moore, William Petty y Leslie Palich (2009), Administración de Pequeñas Empresas: lanzamiento y crecimiento de iniciativas emprendedoras. Cengage Learning Editores; México.

McGregor, Judy, David Tweed y Richard Pech (2004), "Human capital in the new economy: devil's bargain?", Journal of Intellectual Capital, 5, pp. 153-164.

Melo, Ligia Inés y Dora Esther Fonseca (2014), "Descripción y análisis de la gerencia en mipymes agroindustriales del departamento de Boyacá, Colombia", Acta Agronómica, 63 (4), pp. 297-310.

Mujica, Alfonso (2018), "Nuevos requisitos para el perfil del ejecutivo actual", La educación superior y el mercado laboral. <10.31619/caledu.n16.430>. Puértolas, Miguel Ángel (2016), "Inseguridad, razón de Mipymes para no crecer: INEGI”. Milenio, pp. 1-4. http://www.milenio.com/negocios/Inseguridadrazon_de_Mipymes_para_no_crecer-_Inegi_0_775122518.html.

Ramírez, Luz Marina (2005), "Estrategias para desarrollar competencias gerenciales", Revista EAN, 54, pp. 183-204.

Rojas, Mariano, Humberto Angulo e Irene Velázquez (2000), "Rentabilidad de la inversión en capital humano en México", Economía Mexicana, 9(2), pp. 113-142.

Sáenz-Vota, C. y A. Ávila-Arce (2016), "Microempresas y capital humano: un 
análisis para México", Ra Ximhai, 12(4), pp. 183-195.

Solórzano Acuña, Leoncio (2008), "Prospectiva de las competencias gerenciales en la gestión pública", Revista de Investigación en Psicología, 11(2), p. 250.

Tobón, Sergio (2009), Formación basada en competencias. Pensamiento complejo, diseño curricular y didáctica, Editorial CIFE, Barcelona.

Varela, Rodrigo (2002), Innovación empresarial: arte y ciencia en la creación de empresas. Pearson Educación, Colombia.

Walo, Michael (2000), The contribution of internship in Developing IndustryRelevant Management Competencies in Tourism and Hospitality Graduates [Tesis inédita]. Southern Cross University, Australia 